\title{
Some Ferns from the Mountainous Regions of Formosa.
}

(Continued from p. 34.)

\author{
By \\ B. Hay ata \\ Lecturer in Botany, in the College of Science, \\ Imperial University of Tökyō.
}

POLYPODIUM LINN.

Polypodium lineare Thunb.; Matsum. et Hayata, 1.c. p. 632.

Hab. Kagi: Tappansha, (No. 1860) ; in monte Morrison, ad 7000 ped. alt., (No. 1864), leg. T. Kawakami et U. Mori, Oct. 1906.

Polypodium Loxogramme Mett.; Matsum. et Hayata, 1.c. p. 633.

HaB. in monte Morrison, ad 7000 ped. alt., leg. T. KaWAKAMI et U. Mori, Oct. 1606, (No. 1864).

Polypodium cucullatum Nees.; Hooker, Sp. Fil. IV. p. 176 ; Hooker et BaKfr, Syn. Fil. p. 324 ; Christ, Farnk. d. Erd. p. 80, f. 211 ; Diels, Nat. Pfl.fam. p. 309; Copel. Polyp. Philip. p. 120.

Hab. Taitō : Shinsuii, leg. T. Kawakami et U. Mori, April. 1907, (No. 2506).

Distrib. Ceylon, Philippines, Malaisia, Fiji, North Caledonia.

Polypodium formosanum Baker; Matsum. et Hayata, 1. c. p. 630 . 
Hab. in monte Morrison, ad 7000 ped. alt., leg. T. KawAKAMI et U. Mori, Oct. 1906, (No. 1868).

Polypodium irioides Lam.; Matsum. et Hayata, 1. c. p. 632.

Hab. in monte Chōran, leg. G. Nakahara, Aug. 1905, (No. $289)$; Toroku : Hōōsan, leg. T. Kawakami et U. Mori, Nov. 1906, (No. 2340) ; Shintiku : Goshizan, leg. T. Kawakami, Dec. 1906, (No. 2405).

Polypodium Kawakamii Hayata, sp. nov. Rhizomata breve repentia, squamulis majusculis stramineis ovatis supra basin affixis ad medium radiculas emittentibus. Stipites 10-15 cm. longi nudi. Frondes lanceolatæ utrinque attenuatæ circ. $25 \mathrm{~cm}$. longæ $2 \mathrm{~cm}$. latæ integræ rigido-coriaceæ supra nudæ subtus squamulis rotundatis sparce dispersæ, venis venulisque obscuris, areolis copiosis venulis liberis 1-2. Sori magni prominentes non immersi subrotundati vel oblongi prope costam 1-seriatim dispositi supra medium frondum accupantes.

Hab. Toroku : Nanshikiaku, leg. T. Kawakami et U. Mori, Nov. 1906, (No. 2346) ; Arizan, in montis Morrison, leg. G. Nakahara, Nov. 1906.

Somewhat like $P$. Cunninghami Hooker, but differs from it in having rigid texture, very long stipes, and areolas with free veinlets.

Polypodium morrisonense Hayata, sp. nov. Rhizomata lignosa paleis cuspidatis albo-marginatis adpressa. Stipites breves $2 \mathrm{~cm}$. longi erecti. Frondes lanceolatæ circ. $20 \mathrm{~cm}$. longæ $2 \mathrm{~cm}$. latæ apice angustæ obtusæ basi gradatim angustæ ad stipitem brevem abeuntes, margine integræ membranaceæ supra nudæ subtus paleis sparce disprsæ, costis utraque prominulis, venis et venulis valde distinctis, venis ad marginem non attingentibus, areolis inter venas 4-5, venulis liberis $1-2$, venis valde flexuosis. Sori solitarii inter venas laterales.

HaB. in monte Morrison, ad 9000 ped. alt., leg. T. KaWAKAMI et U. Mori, Oct. 1906, (No. 1819). 
Very remarkable for its distinct veins and veinlets, and membranaceous texture.

Polypodium normale Don.; Matsum. et Hayata, 1. c. p. 634.

Hab. Shintiku: Goshizan, leg. T. KawaKami, Dec. 1905, (No. 2404).

Polypodium superficiale Blume; Matsum. et Hayata, 1. c. p. 636 .

HAB. Taitō : Iryokukakusha, leg. T. Kawakami et U. MorI, Dec. 1906, (No. 2360).

Polypodium divaricatum HaYata, sp. nov. Rhizomata repentia, paleis lanceolatis supra basin affixis $4 \mathrm{~mm}$. longis. Stipites erecti $10 \mathrm{~cm}$. longi stramineo-corvini leviter canaliculati lævigato-nudi. Frondes circ. $15 \mathrm{~cm}$. longæ totidem latæ circumscriptione sagittato-cordatæ profunde pinnatifidæ coriaceæ glabræ subtus pallidæ rachis supra planis aut obsolete canaliculatis subtus fortius prominulis rotundatis glaberrimis, venulis utraque prominentibus, lobis utraque latere 6-7 suboppositis $8 \mathrm{~cm}$. longis $1 \frac{1}{2} \mathrm{~cm}$. latis lanceolatis, acuminatis, sinu basi rotundato, margine serrulatis, costis subtus distinctis, venis lateralibus distinctis parallelis, arcolis 4-3 venulis liberis 23 , venulis obscuris marginem fere attingentibus, lobis infimis 2 deflexis, intermediis horizontalibus, superioribus erecto-patentibus, terminali erecto. Sori inter venas laterales solitarii, prope costam tenentes, nunquam ad basin pertingentes.

HaB. in monte Morrison, ad 9000 ped. alt., leg T. KawAKAMI et N. Mori, Oct. 1906, (No. 1871).

Very near to $P$. laciniatum; but differs from that by the serrulate margins of the leaves and coriaceous texture.

Polypodium Lehmanni MetT:; Bedd. Fil. Brit. Ind. t. 260 ; 
Hooker et Baker, Syn. Fil. p. 369 ; Christ, Farn. d. Erd. p. 114.

Hab. Tōzan, in montibus Morrison, leg. G. Nakahara, Dec. 1906.

Polypodium Phymatodes Linn.; Matsum. et Hayata, 1. c. p. 635 .

Hab. Akō: Bangri, leg. G. Nakahara, Jan. 1907.

PJlypodium palmatum BLume? (The identification is rather doubtful).

HaB. in monte Morrison, ad 11000 ped. alt., leg. T. KawAKAMI et U. Mori, Oct. 1906, (No. 1859).

Distrib. Malaisia, the Philippines.

Polypodium pinnatum Hayata, sp. nov. Rhizomata repentia lignosa, paleis fuscis $3 \mathrm{~mm}$. longis acuminatis basi rotundatis supra basin affixis. Stipites $10-12 \mathrm{~cm}$. longi straminei canaliculati. Frondes erectæ $20-25 \mathrm{~cm}$. longæ 15$20 \mathrm{~cm}$. latæ circumscriptione late ovatæ pinnatæ membranaceæ glabræ pallide virides nitidiusculæ, pinnis remotis suboppositis erecto-patentibus, utraque latere 5-6, sessilibus lanceolatis acuminatis circ. $12 \mathrm{~cm}$. longis $1 \frac{1}{2} \mathrm{~cm} .-2 \mathrm{~cm}$. latis basi rotundatis vel oblique cordatis subintegris, costis tenuibus basin glanduliferis subtus promimulis, venis lateralibus rectis suboppositis marginem non attingentibus utraque pagine prominulis, venulis tenerrimis reticulatis, areolis copiosis cum venulis liberis 1-2. Sori totam paginam inferiorem pinnarum occupantes, 2-3 inter venas laterales.

HaB. in monte Morrison, ad 8000 ped. alt., (No. 1873), leg. T. Kawakamr et U. Mori ; Nantō: Musha, (No. 2393), 1906.

Near $P$. angustatum, differs from that by the rounded base of the segments.

Polypodium subauriculatum Blume, Fl. Java. Fil. p. 177, 
t. 83 ; Hooker et Baker, Syn. Fil. p. 344; Christ. Farnk. d. Erd. p. 93; Diels, in Engl. et Prantr. Nat. Pfl.-fam. p. 312 ; Coper. Polyp. Philip. p. 124.

Polypodium pallens Blume, F1. Jav. Fil. p. 178, t. 84, f. 1. Polypodium serratifolium Diels, in Engl. et Prantu. Nat. Pfl.-fam. p. 312.

Hab. Nanto: Musha, leg. T. Kawakami et U. Mori, Aug. 1906, (Nos. 2387 et 2394).

DisTrib. Khasia, Malay peninsula and archipelago.

Polypodium taiwanianum Hayata, sp. nov. Rhizomata repentia flexuosa lignosa, paleis attropurpureis linearibus lanceolatis $5 \mathrm{~mm}$. longis supra basin affixis. Stipites circ. $15 \mathrm{~cm}$. longi, paleis appressiusculis. Frondes latæ circumscriptione triangulares oblongæ circ. $50 \mathrm{~cm}$. longæ $25 \mathrm{~cm}$. latæ pinnatæ membranaceæ supra glabræ, subtus paleis laciniatis minutis sparce dispersæ, pinnis sessilibus linearibus lanceolatis remotiusculis deorsum suboppositis sursum alternis, patentibus acuminatis subintegris, pinnis inferioribus ad basin cordato-auriculatis vel auriculatis, auriculis oblongis $7 \mathrm{~mm}$. longis, costis utrinque distinctis, areolis 2-seriatim dispositis, costalibus magnis cum venulis liberis, marginalibus venulis liberis non instructis. Sori a pinnarum basi usque ad apicem dispositi, solitarii, ad apicem venularum liberarum dispositi.

Hab. Taitō: Taironkōsha, leg. T. Kawakami et U. Mori, Nov. 1906, (No. 2361).

Near P. subauriculatum BLume; but differs from that in having subintire pinnæ and in the arrangement of areolas.

\section{CHEIROPLEURIA PRESL.}

Cheiropleuria bicuspis PREsL. var. integrifolia EAT.; Matsum. et Hayata, 1. c. p. 641.

Hab. Shintiku : Goshizan, leg. T. Kawakami et U. Mori, Dec, 1906, (No. 2389).

The end. 